\title{
Connaissances et usages traditionnels des plantes médicinales du département du haut Nyong
}

\author{
Emmanuel MPONDO MPONDO(1,2), Jean Paul NGENE¹, Léa MPOUNZE SOM¹, Gisèle ETAME LOE1, \\ Philomène Céleste NGO BOUMSONG1, Jacques YINYANG1, Siegfried Didier DIBONG*(1,3). \\ 1 Département des Sciences Pharmaceutiques, Faculté de Médecine et des Sciences Pharmaceutiques, Université de \\ Douala, B.P. 2701 Douala, Cameroun \\ 2 Département de Pharmacotoxicologie et Pharmacocinétique, Faculté de Médecine et des Sciences Biomédicales, \\ Université de Yaoundé I, B.P. 1364 Yaoundé, Cameroun \\ 3 Département de Biologie des Organismes Végétaux, Faculté des Sciences, B.P. 24157 Douala, Cameroun \\ ${ }^{*}$ Auteur de la correspondance : didierdibong@yahoo.fr
}

Original submitted in on $13^{\text {th }}$ February 2017. Published online at www.m.elewa.org on $31^{\text {st }}$ May 2017 https://dx.doi.org/10.4314/jab.v113i1.12

\section{RESUME}

Objectif : Dans le but de contribuer à la connaissance de certaines plantes médicinales et à leurs usages traditionnels sur certains symptômes, une enquête a été réalisée dans trois villages du département du Haut Nyong (région de l'Est).

Matériel et Résultats : L'enquête s'est effectuée auprès de 90 personnes sans distinction d'âge et de sexe. Celles-ci ont été soumises à un questionnaire donnant toutes les informations relatives aux plantes médicinales en langue française ou locale (maka'a). Les données concernant les détails des recettes ont été collectées selon les fiches proposées par la banque de données de médecine traditionnelle et pharmacopée (PHARMEL). Les échantillons ont été récoltés et conservés selon la technique de Schnell. L'identification s'est faite à l'Herbier National du Cameroun. Les données recueillies ont été analysées statistiquement et traitées par le logiciel XLSTAT. Les caractérisations écologiques puis pharmacologiques des espèces végétales ont été réalisées via la revue bibliographique. Quatre-vingt-dix espèces végétales au total ont été identifiées et réparties en 83 genres et 47 familles, la plus représentée a été celle des Euphorbiaceae (10 espèces). Les types phytogéographiques afrotropicales ont été les plus cités. Les sarcochores ont été les types de diaspores dominants et la zoochorie a été le mode de dissémination le plus répandu. Seulement deux plantes recensées montrent une fréquence de citation supérieure ou égale à $50 \%$; il s'agit de Euphorbia hirta $(51 \%)$ et Manihot esculenta $(51 \%)$. Les espèces recensées entrent dans la préparation de 100 recettes. La décoction a été le mode de préparation le plus pratiquée, et la feuille l'organe végétal le plus utilisé. Les plantes citées pourraient contenir pour la plupart des alcaloïdes, les tanins et les flavonoïdes.

Conclusion et Application des résultats : La valorisation de ces plantes et la détermination du profil de santé des populations locales du département du Haut-Nyong nécessite la mise en place des unités de transformations modernes spécialisées dans la fabrication des nouveaux médicaments.

Mots clés : Ethnobotanique, ethno pharmacologique, fréquence de citation, plantes médicinales, métabolites secondaires 


\section{ABSTRACT}

Objective: Plants have been used in traditional medicine for a thousands of years. In order to contribute to the knowledge of some medicinal plants and their traditional uses, for some symptoms, an investigation have been carried out in three subdivisions of Upper Nyong.

Materials and Results: This survey was conducted among 90 individuals regardless of age and sex discrimination. They were subjected to a questionnaire giving all the information relating to medicinal plants in french or local language (maka'a). Data concerning details of revenue collected by the cards offered by traditional medicine database and pharmacopoeia (PHARMEL). Samples were collected and stored according to Schnell technique. The identification was made at the National Herbarium of Cameroon. The collected data were statistically analyzed and processed by the XLSTAT software. Environmental and pharmacological characterization of plant species were carried out via the literature review. Ninety plant species in total were identified and divided into 83 genera and 47 families, most represented was that of Euphorbiaceae (10 species). Afrotropical Phytogeographic types were the most cited. The sarcochores were the dominant types of diaspora. The zoochory was the most common method of dissemination. Only two enumerated plants may show greater than or equal citation frequency to $50 \%$ in the case of Euphorbia hirta (51\%) and Manihot esculenta $(51 \%)$. The recorded species used in the preparation of 100 recipes. The decoction was the most practiced method of preparation, and the leaf the plant organ most used. The listed plants could contain for most, alkaloids, tannins and flavonoids.

Conclusion and application of results: The valuation of these plants and the determination of the local population health profile of Upper Nyong require the establishment of specialized units of modern transformations in the manufacture of new drugs.

Keywords: Ethnobotany, pharmacological ethno, citation frequency, medicinal plants, secondary metabolites

\section{INTRODUCTION}

Les plantes ont été utilisées dans la médecine traditionnelle pendant plusieurs millénaires. La possibilité d'identification des particularités et vertus de chaque plante par sa forme et sa couleur, ont guidé les premiers hommes dans le choix des nouvelles préparations (Newall et al., 1996). Dans les dernières décennies il y a eu un intérêt croissant pour l'étude des plantes médicinales et leur utilisation traditionnelle dans différentes régions du monde. Cette étude s'est avéré une des approches la plus fiable pour la découverte de nouveaux médicaments. De nombreuses populations dans les pays en voie d'émergence pratiquent la médecine traditionnelle à base des plantes. En Europe et en Amérique du nord, un renouveau de l'intérêt envers ces techniques naturelles a été manifeste. Grâce à une meilleure connaissance des mécanismes qui régissent l'action des plantes, mais aussi à la création de standards de sécurité, qualité et de fiabilité en matière de préparation (Patwardhan, 2005). En Afrique, le recours à la médecine et à la pharmacopée traditionnelle est une pratique courante et ancestrale. A l'heure actuelle, près de $80 \%$ de la population africaine a recours aux plantes locales pour se soigner et n'a pas accès aux médicaments modernes. Se soigner par les plantes est connu et pratiquer en Afrique depuis longtemps, car ils exploitent des savoirs transmis oralement de génération en génération (Kanta et al., 2011). L'art de guérir par les plantes médicinales est connu et pratiquer au Cameroun notamment dans le département du HautNyong (région de l'Est) depuis longtemps, par les herboristes, les tradithérapeutes et les villageois. Les connaissances relatives à la médecine traditionnelle sont donc un patrimoine important pour les populations (Muthu et al., 2006). Cependant, en dehors des plantes cultivées, plusieurs plantes sauvages, rudérales ont une grande importance culturelle et économique, pour l'alimentation et les soins. Cette étude consiste donc à l'élaboration d'une enquête ethnobotanique et ethno pharmacologique, afin d'approfondir les connaissances concernant les usages thérapeutiques pratiqués, à l'analyse des résultats concernant les relations existantes entre les espèces médicinales et les catégories de maladies soignées de ce département. 

département du haut Nyong

\section{MATERIEL ET METHODES}

Situation géographique : L'étude a été menée dans trois villages du département du Haut-Nyong (Région de l'Est) : Djamonomine, Motcheboum et Djende (Figure 1). Le Haut-Nyong est un département du Cameroun situé dans la région de l'Est, avec pour chef-lieu Abong-Mbang $\left(3^{\circ} 30^{\prime} 0^{\prime \prime} \mathrm{N}\right.$ et $\left.13^{\circ} 45^{\prime} 0^{\prime \prime} \mathrm{E}\right)$ qui s'étend sur une superficie de $11340 \mathrm{~km}^{2}$. Le climat d'Abong-Mbang est de type forestier avec deux saisons sèches et deux saisons de pluies. La hauteur moyenne annuelle des précipitations varie entre $1600 \mathrm{~mm}$ et $2000 \mathrm{~mm}$. La grande saison des pluies s'étend de mi-août à mi-novembre, tandis que la petite saison des pluies va d'avril à juin. La grande saison sèche se situe entre mi-novembre et mars et la petite saison sèche sévit entre juin et juillet. La température moyenne annuelle oscille entre $23^{\circ} \mathrm{C}$ et $28^{\circ} \mathrm{C}$ avec une forte humidité atmosphérique tout au long de l'année. Le relief de la commune d'Abong-Mbang est légèrement ondulé avec des pics culminant à une altitude ne dépassant pas $600 \mathrm{~m}$. Les sols sont ferralitiques et latéritiques argileux rouges. Étant située dans la forêt équatoriale, les sols de la commune d'Abong-Mbang sont de type forestier très perméable et riche en humus, fertiles et favorables à l'agriculture. Les alluvions et les sols sablonneux sont présents sur les berges du fleuve Nyong et dans les bas-fonds marécageux

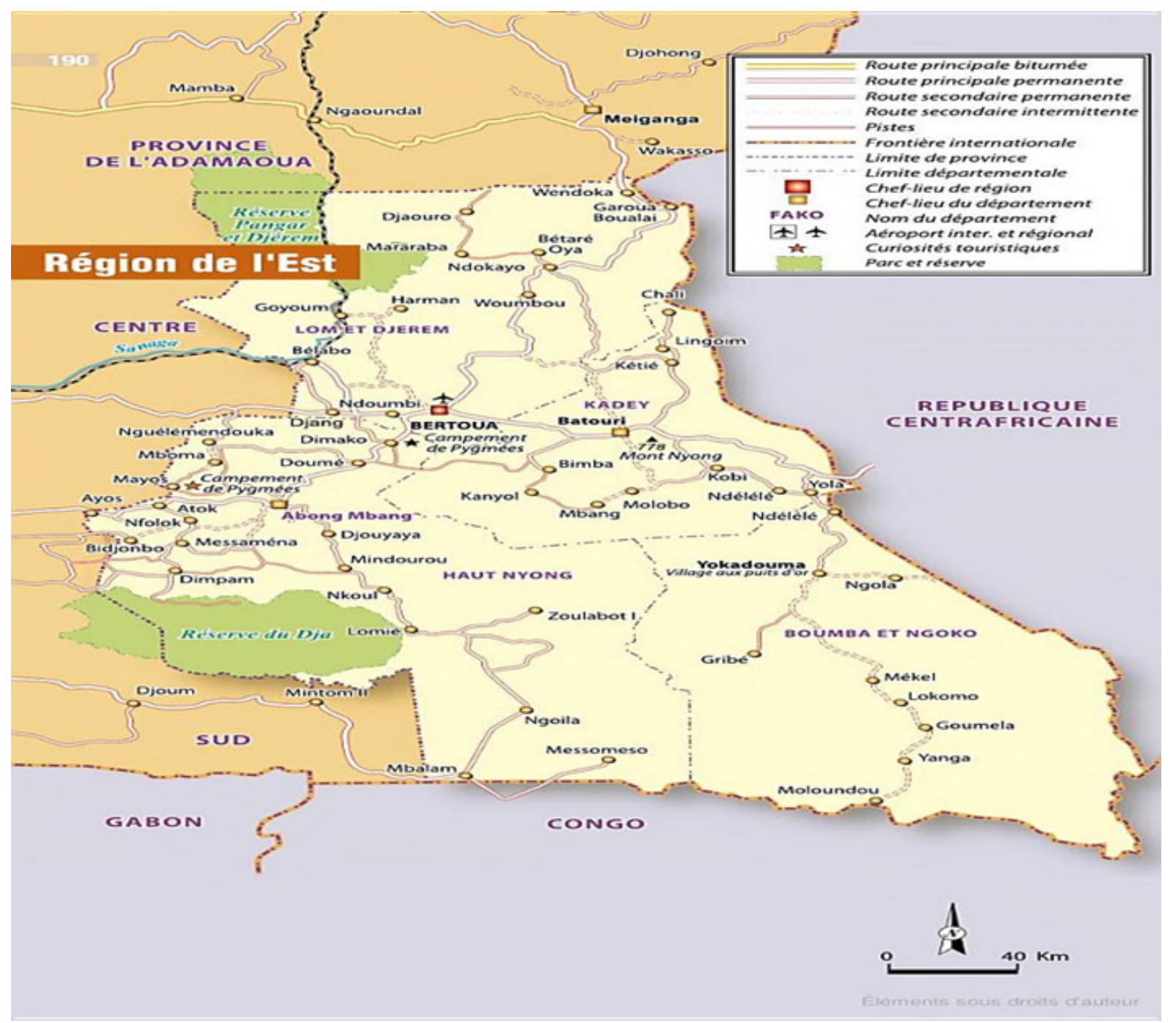

Figure 1 : Carte de localisation de la zone d'étude

L'étude ethnobotanique et ethno pharmacologique s'est réalisée de décembre 2015 à juin 2016. 
Matériel : Le matériel de terrain comporte des fiches d'enquêtes, pour la collecte des informations auprès des enquêtés; des sécateurs, pour la récolte d'échantillons sur le terrain; un appareil photo numérique, pour la prise d'images ; une machette, pour déblayer le passage broussailleux sur le terrain et récolter aussi des organes du végétal ; des papiers journaux, pour la conservation des échantillons récoltés sur le terrain; de l'alcool dilué à $70{ }^{\circ} \mathrm{C}$, pour tremper les échantillons et mieux les conserver; des presses en bois, pour presser et sécher les échantillons; des sacs plastiques, pour le transport du matériel utilisé et des échantillons en vrac c'est-à-dire non encore sélectionnés. Tout ce matériel était nécessaire pour une bonne récolte et une meilleure conservation des échantillons obtenus sur le terrain.

Les plantes sont récoltées à l'état frais dans leur biotope, en s'assurant de la période de récolte de chacune d'elle. La récolte se fait durant la floraison ou la fructification en raison de leur concentration élevée en métabolites secondaires.

\section{Méthodologie}

Enquêtes socio-économiques : L'enquête sur l'usage traditionnel des plantes médicinales a été réalisée parmi les populations de 3 villages du Département du HautNyong. Comme approche utilisée, une visite a été rendue aux enquêtés et un entretien semi structuré leur a été proposé. Les renseignements fournis portaient sur les noms locaux des plantes utilisées, les différentes parties employées comme drogues, les méthodes de récolte, les modes de préparation et d'administration des recettes médicamenteuses. Les propriétés thérapeutiques de ces plantes ont été recensées en utilisant la documentation en rapport avec les travaux

\section{RESULTATS}

Caractéristiques socio-démographiques des enquêtés: Quatre-vingt dix personnes réparties dans trois villages (30 à Djamonomine, 30 à Motcheboum, 30 à Djende) ont été interviewés sur les connaissances et les usages traditionnels des plantes médicinales. La majorité des enquêtés a était constituée des hommes soit 54,55 $\%$. L'âge des enquêtés a varié de 25 à 81 ans répartien 3 classes : les jeunes ] 20-30 ans], adultes [41-50ans] et vieillards [> 50[. $62,22 \%$ ont affirmé avoir reçu leurs savoirs de leurs descendants, $11,11 \%$ ont admis l'avoir appris et $26,66 \%$ de manière héréditaire et empirique (tableau 1).

Diversité taxonomique : L'inventaire floristique a permis d'identifier 47 familles, réparties en 83 genres et 90 espèces. Les familles majoritaires ont été les relatifs au screening phytochimique et l'étude pharmacologique.

Inventaire de la florule: L'Inventaire de la florule consistait à reconnaitre la plante dans son milieu naturelle, ensuite faire une prise de vue à l'aide d'un appareil photo numérique et enfin nommez la plante en langue vernaculaire ou en utilisant les noms communs attribués à ces plantes médicinales.

Identification des espèces : Sur le terrain, les plantes citées en langue vernaculaire ont été reconnues in situ et les échantillons complets ont été récoltés et séchés après aspersion à l'alcool à $70^{\circ} \mathrm{C}$ dont le but était de conserver la coloration des organes végétaux.

$\mathrm{Au}$ laboratoire de biologie des organismes végétaux l'observation des photos et des échantillons des plantes récoltées ont permis de reconnaitre les espèces et de préciser leur genre et leur famille. Cette taxonomie par la suite était confirmée à l'herbier national du Cameroun où les numéros de référence ont été attribués aux espèces végétales recensées.

Description des paramètres biologiques : Les espèces identifiées ont été décrites en types phytogéographiques, types morphologiques et types de diaspores. Cette description a été rendue possible en utilisant la documentation scientifique appropriée et les différents volumes de la flore du Cameroun stockés à l'herbier national du Cameroun.

Analyse des données : Les données de terrain ont été enregistrées sur un tableur Excel, analysées statistiquement et traitées par le logiciel XLSTAT version 11.0.0.28480. L'analyse factorielle des correspondances a été employée pour montrer les variations spatiales des espèces végétales entre les villages.

Euphorbiaceae $11,11 \%$, suivies des Fabaceae et Malvaceae soit $5,55 \%$ chacune et les Rubiaceae $4,44 \%$. Paramètres écologiques des espèces végétales : Les plantes médicinales inventoriées comptent 11 types biologiques dont les plus représentés sont les mégaphanérophytes estimés à $12,22 \%$. Les espèces identifiées comptent 24 types phytogéographiques avec une prédominance d'espèces afro-tropicales $(34,44 \%)$ suivies des espèces guinéennes avec $13,33 \%$.La majorité des plantes recensées sont des arbres $(33,33 \%)$, suivis des arbustes et des herbacées avec chacun 20\%.Le recensement a dénombré 5 types de diaspores avec une majorité pour les sarcochores $(66,66 \%)$, suivis des sclérochores avec $11,11 \%$. 


\section{Mpondo et al., J. Appl. Biosci. 2017 Connaissances et usages traditionnels des plantes médicinales du}

département du haut Nyong

Tableau 1: Caractéristiques socio-démographiques des enquêtés interviewés ( $\mathrm{n}=90)$

\begin{tabular}{cc}
\hline Caractéristiques & Fréquence (\%) \\
Genre & \\
Femmes & $45,55 \%$ \\
Hommes & $54,55 \%$ \\
& \\
Age & \\
]20-30] & $22,22 \%$ \\
{$[31-40]$} & $21,11 \%$ \\
{$[41-50]$} & $7,77 \%$ \\
$>50$ & $14,44 \%$ \\
& \\
Nature d'acquisition des connaissances & \\
Héréditaire & $62,22 \%$ \\
Empirique & $11,11 \%$ \\
Héréditaire et Empirique & $26,66 \%$ \\
& \\
Répartition par village & \\
Djamonomine & $33,33 \%$ \\
Motcheboum & $33,33 \%$ \\
Djende & $33,33 \%$ \\
\hline
\end{tabular}

Modes de préparation, modes d'administration, recettes des plantes, maladies et symptômes traités: Le mode de préparation majoritaire utilisé pour les recettes des espèces végétales est la décoction $(38,04 \%)$, suivie de la trituration avec $31,95 \%$. Des sept modes retenus, la plus employée par les villageois est la voie orale avec $67,77 \%$. L'étude ethnopharmacologique a permis de distinguer 90 recettes à base de plantes médicinales accompagnées de leur mode de préparation et d'administration. Le facteur de citation ( $\mathrm{Fc}$ ) calculé est compris entre $3,33 \%$ et $51 \%$ (Tableau 2).

Propriétés thérapeutiques des métabolites secondaires: La recherche bibliographique a montré que toutes les espèces végétales recensées sont riches en métabolites secondaires. Les résultats montrent une prédominance de certaines plantes riches en alcaloïdes $(35,55 \%)$, tanins $(23,33 \%)$ et flavonoïdes $(16,66 \%)$. En effet les plantes à alcaloïdes sont utilisées comme antalgique, antiparasitaire, antipyrétique, laxatif, antiulcéreux, antitussif, anti hémorroïdaires. Les plantes à tanins sont utilisées comme antiparasitaire, antalgique, anti diarrhéique, antalgique et les plantes à flavonoïdes sont utilisées comme laxatif, antalgique et antipyrétique. Les propriétés thérapeutiques de ces plantes sont en accord avec les recettes données par les enquêtés d'où l'importance d'une valorisation de la pharmacopée traditionnelle du département du Haut Nyong en vue de la mise en place des unités de transformations modernes spécialisées dans la fabrication des médicaments. 
Tableau 2 : Tableau des recettes de plantes médicinales recensées

\begin{tabular}{|c|c|c|c|c|c|c|c|}
\hline Famille & $\begin{array}{l}\text { Noms } \\
\text { scientifique }\end{array}$ & $\begin{array}{l}\text { Plantes } \\
\text { associées }\end{array}$ & $\begin{array}{l}\text { Organes } \\
\text { utilisés }\end{array}$ & $\begin{array}{l}\text { Fréquenc } \\
\text { e de } \\
\text { citation }\end{array}$ & $\begin{array}{l}\text { Maladies et } \\
\text { symptômes }\end{array}$ & Mode de préparation & $\begin{array}{l}\text { Mode } \\
\text { d'administration, } \\
\text { posologie, durée du } \\
\text { traitement }\end{array}$ \\
\hline 1-Menispermaceae & $\begin{array}{l}\text { Cissampelos } \\
\text { owariensis }\end{array}$ & Aucune & Feuilles & 3,33 & Hémorragie & $\begin{array}{l}\text {-Trituration des feuilles } \\
\text { dans un demi-litre } \\
\text { d'eau. }\end{array}$ & $\begin{array}{l}\text { Boire la totalité (demi- } \\
\text { litre) par jour pendant } \\
1 \text { mois }\end{array}$ \\
\hline 2-Myristiceae & $\begin{array}{l}\text { Pycnanthus } \\
\text { angolensis }\end{array}$ & Aucune & $\begin{array}{l}\text { Écorce et } \\
\text { sève }\end{array}$ & 11,11 & $\begin{array}{l}\text { Infection respiratoire, } \\
\text { gingivite }\end{array}$ & $\begin{array}{l}\text {-Décoction dans } 2 \text { I } \\
\text { d'eau. }\end{array}$ & $\begin{array}{l}\text { Boire 1 verre matin et } \\
\text { soir pendant } 1 \\
\text { semaine }\end{array}$ \\
\hline 3-Sapindaceae & Paullinia pinnata & Aucune & Feuilles & 12,22 & $\begin{array}{l}\text { Pneumonie chez les } \\
\text { enfants }\end{array}$ & $\begin{array}{l}\text {-Décoction ou } \\
\text { trituration dans } 21 \\
\text { d'eau puis filtré }\end{array}$ & $\begin{array}{l}\text { Donner une cuillère à } \\
\text { café chaque } 3 \mathrm{~h} \\
\text { pendant } 1 \text { semaine }\end{array}$ \\
\hline 4-Rubiaceae & Tarenna sp. & Aucune & Tige & 10 & Rougeole, varicelle & $\begin{array}{l}\text {-Trituration des tiges } \\
\text { puis mettre dans un } \\
\text { litre d'eau, filtrer } \\
\text { ensuite ajouter du vin } \\
\text { de palme }\end{array}$ & $\begin{array}{l}\text { Une purge par jour, la } \\
\text { durée du traitement } \\
\text { dépend de la gravite } \\
\text { de l'infection }\end{array}$ \\
\hline 5-Costaceae & Costus afer & Aucune & Écorces & 16,66 & Anémie, varicelle & $\begin{array}{l}\text {-Macération pendant } \\
2 \mathrm{~h}\end{array}$ & $\begin{array}{l}\text { Boire } 1 \text { verre par jour } \\
\text { pendant } 1 \text { mois }\end{array}$ \\
\hline 6-Connaraceae & Cnestis corniculata & Aucune & Tige & 7,77 & Ocquet, & $\begin{array}{l}\text {-Macération dans } 2 \mathrm{I} \\
\text { d'eau pendant } 2 \mathrm{~h}\end{array}$ & $\begin{array}{l}1 \text { verre matin et soir } \\
\text { par jour jusqu'à } \\
\text { terminer le macéré, } 1 \\
\text { cataplasme matin et } \\
\text { soir }\end{array}$ \\
\hline 7-Combretaceae & $\begin{array}{l}\text { Combretum } \\
\text { micranthum }\end{array}$ & Aucune & Feuilles & 7,77 & Paludisme & $\begin{array}{l}\text {-Decoction dans 3I } \\
\text { d'eau }\end{array}$ & $\begin{array}{l}1 \text { verre matin et soir } \\
\text { pendant } 4 \text { jours }\end{array}$ \\
\hline 8-Euphorbiaceae & $\begin{array}{l}\text { Manniophyton } \\
\text { fulvum }\end{array}$ & Aucune & $\begin{array}{l}\text { Jeunes } \\
\text { feuilles }\end{array}$ & 22,22 & Diarrhee & $\begin{array}{l}\text {-Trituration des feuilles } \\
\text { légèrement chauffées }\end{array}$ & $\begin{array}{l}\text { Prise unique par jour } \\
\text { pendant } 3 \text { jours }\end{array}$ \\
\hline 9-Euphorbiaceae & $\begin{array}{l}\text { Macaranga } \\
\text { spinosa }\end{array}$ & Aucune & Feuilles & 12,22 & Infection vaginale & -Trituration des feuilles & $\begin{array}{l}\text { Une pâte au coucher } \\
\text { pendant } 10 \text { jours }\end{array}$ \\
\hline 10-Euphorbiaceae & Bridelia micrantha & $\begin{array}{l}\text { Seule ou } \\
\text { chieng }\end{array}$ & Écorces & 16,66 & $\begin{array}{l}\text { Lombalgie, } \\
\text { inssufisance renale }\end{array}$ & $\begin{array}{l}\text {-Macération dans } 4 \mathrm{I} \\
\text { d'eau pendant } 2 \mathrm{~h}\end{array}$ & $\begin{array}{l}\text { Boire } 1 \text { verre par jour } \\
\text { ou une purge par jour }\end{array}$ \\
\hline 11-Araliaceae & Polyscias fulva & Aucune & Écorces & 11,11 & Toux & -Décoction des & 1 verre matin et soir \\
\hline
\end{tabular}




\begin{tabular}{|c|c|c|c|c|c|c|c|}
\hline & & & & & & $\begin{array}{l}\text { écorces dans } 2 \text { litre } \\
\text { d'eau, mettre } \\
\text { légèrement de sel et } \\
\text { du petit piment rouge. } \\
\text { mélanger, filtrer }\end{array}$ & $\begin{array}{l}\text { jusqu'à ce que la toux } \\
\text { finisse }\end{array}$ \\
\hline 12-Anacadiaceae & Mangifera foetida & Aucune & Écorces & 13,33 & Carie dentaire & $\begin{array}{l}\text {-Décoction dans } 3 \\
\text { litres d'eau puis filtré }\end{array}$ & $\begin{array}{l}\text { Mettre un quart de } \\
\text { verre du décocté dans } \\
\text { la bouche, attendre } 3 \\
\text { mn puis avaler matin } \\
\text { et soir }\end{array}$ \\
\hline 13-Anacadiaceae & Sorindeia sp. & Aucune & Écorces & 13,33 & Toux & $\begin{array}{l}\text {-Décoction dans } 5 \text { I } \\
\text { d'eau ensuite filtrer }\end{array}$ & $\begin{array}{l}\text { Boire } 1 \text { verre par jour } \\
\text { pendant } 2 \text { a } 3 \text { jours }\end{array}$ \\
\hline 14-Acanthaceae & $\begin{array}{l}\text { Eremomastax } \\
\text { speciosa }\end{array}$ & Aucune & $\begin{array}{l}\text { Feuilles, } \\
\text { racines }\end{array}$ & $16,66 \mathrm{em}$ & $\begin{array}{l}\text { Contipation chez les } \\
\text { bebe, erytheme } \\
\text { fessier }\end{array}$ & $\begin{array}{l}\text {-Décoction, filtrer } \\
\text { ensuite purger le bébé }\end{array}$ & $\begin{array}{l}\text { Une purge par jour } \\
\text { pendant } 3 \text { a } 4 \text { jours }\end{array}$ \\
\hline $\begin{array}{l}\text { 15-Commelina } \\
\text { ceae }\end{array}$ & Floscapa africana & Aucune & Feuilles & 11,11 & Inssufisance renale & $\begin{array}{l}\text {-Décoction dans } 31 \\
\text { d'eau puis filtrer }\end{array}$ & $\begin{array}{l}\text { Boire } 2 \text { verres par jour } \\
\text { pendant } 4 \text { jours }\end{array}$ \\
\hline 16-Meliaceae & Guarea sp. & Aucune & $\begin{array}{l}\text { Feuilles, } \\
\text { ecorces }\end{array}$ & 16,66 & Syphillis, lombalgie & $\begin{array}{l}\text {-Décoction des feuilles } \\
\text { et des écorces, filtré } \\
\text { puis purger }\end{array}$ & $\begin{array}{l}\text { Une purge après un } \\
\text { jour pendant } 2 \\
\text { semaines }\end{array}$ \\
\hline 17-Arecaceae & $\begin{array}{l}\text { Rhektophyllum } \\
\text { mirabile }\end{array}$ & Aucune & $\begin{array}{l}\text { Tubercule } \\
\mathrm{s}\end{array}$ & 15,55 & Furoncle, abces & $\begin{array}{l}\text {-Trituration des } \\
\text { tubercules puis poser } \\
\text { sur le furoncle ou } \\
\text { l'abcès et attacher }\end{array}$ & $\begin{array}{l}\text { Poser la pate matin et } \\
\text { soir jusqu'à ce que le } \\
\text { furoncle ou l'abcès } \\
\text { finisse }\end{array}$ \\
\hline $\begin{array}{l}\text { 18-Melastomata } \\
\text { ceae }\end{array}$ & $\begin{array}{l}\text { Tristemma } \\
\text { mauritianum }\end{array}$ & Aucune & Feuilles & 10 & Anemie & $\begin{array}{l}\text {-Décoction des } \\
\text { feuilles dans } 2 \text { I d'eau, } \\
\text { filtrer }\end{array}$ & $\begin{array}{l}\text { Boire } 1 \text { verre matin et } \\
\text { soir pendant } 1 \text { mois }\end{array}$ \\
\hline 19-Marantaceae & $\begin{array}{l}\text { Marantochloa } \\
\text { comorensis }\end{array}$ & Aucune & Tige & 13,33 & Helmethiases & $\begin{array}{l}\text {-Trituration de la tige } \\
\text { puis avaler son jus }\end{array}$ & $\begin{array}{l}\text { Mâcher la tige et } \\
\text { avaler son jus chaque } \\
\text { jour jusqu'à ce que le } \\
\text { mal finisse }\end{array}$ \\
\hline 20-Euphorbiaceae & Euphorbia hirta & $\begin{array}{l}\text { Feuilles de } \\
\text { goyavier }\end{array}$ & Feuilles & 51 & $\begin{array}{l}\text { Dysenteries } \\
\text { amibiennes, diarrhee }\end{array}$ & $\begin{array}{l}\text {-Décoction des feuilles } \\
\text { avec celles du } \\
\text { goyavier ajouté un peu } \\
\text { de sel }\end{array}$ & $\begin{array}{l}\text { Demi verre matin et } \\
\text { soir pendant } 5 \text { jours }\end{array}$ \\
\hline 21-Nyctaginaceae & Boerrhavia repens & Aucune & Feuilles & 11,11 & Toux chez les enfants & -Expression Des & Mettre 2 gouttes dans \\
\hline
\end{tabular}


Mpondo et al., J. Appl. Biosci. 2017 Connaissances et usages traditionnels des plantes médicinales du département du haut Nyong

\begin{tabular}{|c|c|c|c|c|c|c|c|}
\hline & & & & & & feuilles, ajouter du sel. & chaque narine \\
\hline 22-Moraceae & Ficus sur & Aucune & Feuilles & 16,66 & $\begin{array}{l}\text { Infection de la peau } \\
\text { chez les enfants }\end{array}$ & $\begin{array}{l}\text {-Trituration des } \\
\text { feuilles, .mélanger et } \\
\text { oindre l'enfant }\end{array}$ & $\begin{array}{l}\text { Oindre l'enfant matin } \\
\text { et soir après son bain } \\
\text { jusqu'à ce que } \\
\text { l'infection finisse }\end{array}$ \\
\hline 23-Fabaceae & Senna alata & Aucune & Feuilles & 33,33 & Fievre typhoide, datre & $\begin{array}{l}\text {-décoction ou } \\
\text { trituration des feuilles. }\end{array}$ & $\begin{array}{l}\text { Boire } 1 \text { verre par jour } \\
\text { pour la jaunisse, frotter } \\
\text { sur la peau matin et } \\
\text { soir après son bain }\end{array}$ \\
\hline 24-Malvaceae & $\begin{array}{l}\text { Sida } \\
\text { rhombifullium. }\end{array}$ & Aucune & Feuilles & 11,11 & $\begin{array}{l}\text { Ulcères gastriques, } \\
\text { hypertension artérielle }\end{array}$ & -Macération & $\begin{array}{l}1 \text { verre matin et soir } \\
\text { pendant une semaine }\end{array}$ \\
\hline 25-Dillenaceae & $\begin{array}{l}\text { Tetracera } \\
\text { cf.alnifolia }\end{array}$ & Aucune & Feuilles & 11,11 & Traumatisme de l'oeil & $\begin{array}{l}\text {-Trituration des } \\
\text { feuilles, mettre dans } \\
\text { l'œil blesser }\end{array}$ & $\begin{array}{l}2 \text { gouttes dans l'œil } \\
\text { blessé jusqu'à } \\
\text { guérison }\end{array}$ \\
\hline 26-Euphorbiaceae & $\begin{array}{l}\text { Alchornea } \\
\text { floribunda }\end{array}$ & Aucune & Feuilles & 22,22 & Helminthiases & $\begin{array}{l}\text {-Trituration des } \\
\text { feuilles, avaler son jus. }\end{array}$ & $\begin{array}{l}\text { Le faire chaque matin } \\
\text { et soir jusqu'à ce que } \\
\text { le mal finisse }\end{array}$ \\
\hline 27-Rutaceae & Zanthoxyllum sp. & Aucune & Écorce & 16,66 & Douleur musculaire & $\begin{array}{l}\text {-Trituration de l'écorce } \\
\text { raclée sur la partie } \\
\text { douloureuse }\end{array}$ & $\begin{array}{l}\text { Masser matin et soir } \\
\text { jusqu'à ce que la } \\
\text { douleur finisse }\end{array}$ \\
\hline 28-Rubiaceae & Uncaria sp. & Aucune & $\begin{array}{l}\text { Jeunes } \\
\text { feuilles }\end{array}$ & 11,11 & $\begin{array}{l}\text { Fermeture lente de la } \\
\text { fontanelle }\end{array}$ & $\begin{array}{l}\text {-Trituration des } \\
\text { feuilles, chauffée } \\
\text { légèrement }\end{array}$ & $\begin{array}{l}1 \text { cataplasme matin et } \\
\text { soir pendant } 2 \\
\text { semaines }\end{array}$ \\
\hline 29-Arecaceae & Elaeis guineensis & Aucune & $\begin{array}{l}\text { Jeunes } \\
\text { feuilles }\end{array}$ & 11,11 & Fievre thyphoide & $\begin{array}{l}\text {-Trituration des } \\
\text { feuilles, filtrer et boire. }\end{array}$ & $\begin{array}{l}\text { Prise unique par jour } \\
\text { pendant } 3 \text { jours }\end{array}$ \\
\hline 30-Fabaceae & $\begin{array}{l}\text { Tetrapleura } \\
\text { tetraptera }\end{array}$ & Aucune & Fruit & 11,11 & Dysménorrhées & $\begin{array}{l}\text {-décoction du fruit, } \\
\text { filtrer }\end{array}$ & $\begin{array}{l}\text { Une purge matin et } \\
\text { soir }\end{array}$ \\
\hline 31-Sterculiaceae & $\begin{array}{l}\text { Cola } \\
\text { chlamydantha }\end{array}$ & Aucune & Écorces & 11,11 & $\begin{array}{l}\text { Faiblesse sexuelle } \\
\text { chez l'homme }\end{array}$ & -Maceration & $\begin{array}{l}\text { Boire comme son eau } \\
\text { jusqu'à atteindre } \\
\text { l'efficacité }\end{array}$ \\
\hline 32-Euphorbiaceae & Alchornea laxiflora & Aucune & Feuilles & 11,11 & Abces & -Trituration & $\begin{array}{l}\text { matin et soir jusqu'à } \\
\text { ce que le pus sorte }\end{array}$ \\
\hline 33-Cecropiaceae & $\begin{array}{l}\text { Myrianthus } \\
\text { arboreus }\end{array}$ & Aucune & Feuilles & 22,22 & Hernie ombilicale & -Maceration & $\begin{array}{l}\text { Une purge par jour } \\
\text { jusqu'à ce que } \\
\text { l'ombrine diminue }\end{array}$ \\
\hline 34-Lamiaceae & Clerodendrum sp. & Aucune & Écorces & 14,44 & Splenomegalie & - Décoction, trituration & Une purge par jour \\
\hline
\end{tabular}


Mpondo et al., J. Appl. Biosci. 2017 Connaissances et usages traditionnels des plantes médicinales du département du haut Nyong

\begin{tabular}{|c|c|c|c|c|c|c|c|}
\hline & & & & & & & $\begin{array}{l}\text { jusqu'à diminution de } \\
\text { la rate }\end{array}$ \\
\hline 35-Lamiaceae & Vitex doniana & Aucune & Écorces & 22,22 & Paludisme & -Decoction & $\begin{array}{l}\text { Un verre matin et soir } \\
\text { pendant } 4 \text { jours }\end{array}$ \\
\hline 36-Cecropiaceae & $\begin{array}{l}\text { Musanga } \\
\text { cecropioides }\end{array}$ & Aucune & Racines & 16,66 & $\begin{array}{l}\text { Purification du lait } \\
\text { maternel }\end{array}$ & $\begin{array}{l}\text { - Recueillir chaque } \\
\text { matin l'eau des } \\
\text { racines et boire } \\
\text { comme son eau }\end{array}$ & $\begin{array}{l}\text { Boire jusqu'à atteindre } \\
\text { l'efficacité }\end{array}$ \\
\hline 37-Apocynaceae & $\begin{array}{l}\text { Tabernaemontana } \\
\text { sp. }\end{array}$ & Aucune & Tige, sève & 26,66 & $\begin{array}{l}\text { Blessures } \\
\text { superficielles de la } \\
\text { peau }\end{array}$ & -Trituration & $\begin{array}{l}\text { Pansement chaque } \\
\text { matin et soir }\end{array}$ \\
\hline 38-Rubiaceae & Diodia sarmentosa & Aucune & Feuilles & 11,11 & Douleur abdominale & -Macération & $\begin{array}{l}\text { Demi litre par jour } \\
\text { jusqu'à ce que la } \\
\text { douleur finisse }\end{array}$ \\
\hline 39-Acanthaceae & $\begin{array}{l}\text { Acanthus } \\
\text { montanus }\end{array}$ & Aucune & Racines & 11,11 & Abces & -Trituration & $\begin{array}{l}\text { Poser chaque matin et } \\
\text { soir jusqu'a a la } \\
\text { guérison }\end{array}$ \\
\hline 40-Loacea & Setaria barbata & Aucune & Feuilles & 33,33 & $\begin{array}{l}\text { Diarrhées chez les } \\
\text { enfants }\end{array}$ & $\begin{array}{l}\text {-Macération dans un } \\
\text { demi-verre d'eau et } \\
\text { boire }\end{array}$ & $\begin{array}{l}\text { Boire un demi verre } \\
\text { par jour jusqu'a arrêt } \\
\text { de la diarrhée }\end{array}$ \\
\hline 41-Cucurbitaceae & Momordica foetida & Aucune & Tige & 27,77 & $\begin{array}{l}\text { Faiblesse sexuelle } \\
\text { chez l'homme }\end{array}$ & $\begin{array}{l}\text {-Macération dans 1 I } \\
\text { de vin de palme, fitrer, }\end{array}$ & $\begin{array}{l}\text { Boire } 1 \text { verre matin et } \\
\text { soir jusqu'à atteindre } \\
\text { l'efficacité }\end{array}$ \\
\hline 42-Fabaceae & Erythrophloem sp. & Aucune & Tige & 16,66 & Ulcère gastrique & $\begin{array}{l}\text {-Macération dans } 2 \text { I } \\
\text { d'eau, filtrer puis boire }\end{array}$ & $\begin{array}{l}\text { Boire } 1 \text { verre matin et } \\
\text { soir jusqu'à ce que la } \\
\text { douleur finisse }\end{array}$ \\
\hline 43-Poaceae & $\begin{array}{l}\text { Cymbopogon } \\
\text { citratus }\end{array}$ & Aucune & Feuilles & 22,22 & Fièvre & $\begin{array}{l}\text {-Décoction, boire la } \\
\text { tisane chaude }\end{array}$ & $\begin{array}{l}\text { Un verre matin et soir } \\
\text { pendant } 4 \text { jours }\end{array}$ \\
\hline 44-Cucurbitaceae & $\begin{array}{l}\text { Momordica } \\
\text { cissoides }\end{array}$ & Aucune & Feuilles & 22,22 & Hemorroides, otites & -Décoction & \\
\hline 45-Asteraceae & Mikania scandens & Aucune & Racines & 10 & Dysménorrhée & -Decoction & $\begin{array}{l}\text { Une purge le premier } \\
\text { jour des règles }\end{array}$ \\
\hline 46-Dillenraceae & $\begin{array}{l}\text { Tetracera cf. } \\
\text { mayumbensis }\end{array}$ & Aucune & Écorces & 11,11 & Paludisme & -Decoction & $\begin{array}{l}\text { Boire } 2 \text { verres matin et } \\
\text { soir pendant } 4 \text { jours }\end{array}$ \\
\hline 47-Combretaceae & Combretum sp. & Aucune & $\begin{array}{l}\text { Jeunes } \\
\text { feuilles }\end{array}$ & 11,11 & Infection vaginale & -Trituration & $\begin{array}{l}\text { Une petite pâte au } \\
\text { coucher pendant } 10\end{array}$ \\
\hline
\end{tabular}


Mpondo et al., J. Appl. Biosci. 2017 Connaissances et usages traditionnels des plantes médicinales du département du haut Nyong

\begin{tabular}{|c|c|c|c|c|c|c|c|}
\hline & & & & & & & jours \\
\hline 48-Euphorbiaceae & $\begin{array}{l}\text { Alchornea } \\
\text { floribunda }\end{array}$ & Aucune & $\begin{array}{l}\text { Tubercule } \\
\mathrm{s}\end{array}$ & 14,44 & Panaris des pieds & $\begin{array}{l}\text {-Décoction dans } 31 \\
\text { d'eau, rendre tiède } \\
\text { puis tremper les pieds } \\
\text { malade dans le } \\
\text { récipient }\end{array}$ & $\begin{array}{l}\text { Tremper le pied } \\
\text { malade matin et soir } \\
\text { pendant } 10 \mathrm{mn} \\
\text { jusqu'a guérison }\end{array}$ \\
\hline 49-Euphorbiaceae & $\begin{array}{l}\text { Tetrorchidium } \\
\text { didymostemon }\end{array}$ & Aucune & Feuilles & 13,33 & $\begin{array}{l}\text { Constipation } \\
\text { chronique }\end{array}$ & $\begin{array}{l}\text {-Macération des } \\
\text { feuilles chauffées puis } \\
\text { ajouter du vin de } \\
\text { palme, mélanger puis } \\
\text { filtrer }\end{array}$ & Boire très tôt le matin \\
\hline 50-Rubiaceae & $\begin{array}{l}\text { Craterispermum } \\
\text { cf. aristatum }\end{array}$ & Aucune & Racines & 8,88 & $\begin{array}{l}\text { Blessures } \\
\text { superficielles de la } \\
\text { peau }\end{array}$ & - Trituration & $\begin{array}{l}\text { Poser la pâte sur la } \\
\text { blessure chaque après } \\
2 \text { jours }\end{array}$ \\
\hline 51-Zingiberaceae & Aframomum sp. & Aucune & Feuilles & 22,22 & Carie dentaire & $\begin{array}{l}\text {-Macération dans un } \\
\text { peu d'eau, mettre } \\
\text { dans un entonnoir en } \\
\text { feuille }\end{array}$ & $\begin{array}{l}\text { Mettre } 2 \text { gouttes sur } \\
\text { chaque dent malade }\end{array}$ \\
\hline 52-Clusiaceae & $\begin{array}{l}\text { Allanblackia } \\
\text { floribunda }\end{array}$ & Aucune & Écorces & 8,88 & Toux chez l'adulte & $\begin{array}{l}\text {-Décoction, filtrer et } \\
\text { boire tiède }\end{array}$ & $\begin{array}{l}\text { Boire } 1 \text { verre matin et } \\
\text { soir pendant une } \\
\text { semaine }\end{array}$ \\
\hline 53-Moraceae & Ficus exasperata & Aucune & Feuilles & 6,66 & Fièvre thyphoide & $\begin{array}{l}\text {-Macération + du vin } \\
\text { de palme, }\end{array}$ & $\begin{array}{l}\text { Boire } 1 \text { verre par jour } \\
\text { pendant } 4 \text { jours }\end{array}$ \\
\hline 54-Acanthaceae & $\begin{array}{l}\text { Pseuderanthemum } \\
\text { tunicatum }\end{array}$ & Aucune & Feuilles & 7,77 & Inflammation cutanee & $\begin{array}{l}\text {-Trituration, mélanger } \\
\text { à de l'huile de palmiste }\end{array}$ & $\begin{array}{l}\text { Oindre la peau après } \\
\text { chaque bain matin et } \\
\text { soir }\end{array}$ \\
\hline 55-Clusiaceae & Garcinia kola & Aucune & $\begin{array}{l}\text { Fruits, } \\
\text { ecorces }\end{array}$ & 8,88 & $\begin{array}{l}\text { ulcere gastrique, } \\
\text { diabete }\end{array}$ & -Décoction & Un verre matin et soir \\
\hline 56-Hernandiaceae & Illigera pentaphylla & Aucune & Feuilles & 22,22 & Anorexie & $\begin{array}{l}\text {-Trituration puis mettre } \\
\text { dans la nourriture }\end{array}$ & $\begin{array}{l}2 \text { à } 3 \text { fois par jour } \\
\text { pendant } 4 \text { jours }\end{array}$ \\
\hline 57-Rubiaceae & $\begin{array}{l}\text { Schumanniophyto } \\
n \text { magnificum }\end{array}$ & Aucune & Racines & 27,77 & Otites & $\begin{array}{l}\text {-Trituration des } \\
\text { racines, ajouter de } \\
\text { l'huile de palmiste, } \\
\text { chauffer légèrement. }\end{array}$ & $\begin{array}{l}\text { Presser le liquide } \\
\text { obtenu } 2 \text { gouttes dans } \\
\text { l'oreille qui coule matin } \\
\text { et soir }\end{array}$ \\
\hline
\end{tabular}


Mpondo et al., J. Appl. Biosci. 2017 Connaissances et usages traditionnels des plantes médicinales du département du haut Nyong

\begin{tabular}{|c|c|c|c|c|c|c|c|}
\hline 58-Malvaceae & $\begin{array}{l}\text { Gossypium } \\
\text { barbadense }\end{array}$ & Aucune & Feuilles & 33,33 & Fievre typhoide & $\begin{array}{l}\text {-Trituration des feuilles } \\
\text { dans } 2 \text { I d'eau }\end{array}$ & $\begin{array}{l}\text { Boire } 1 \text { verre matin- } \\
\text { midi-soir }\end{array}$ \\
\hline 59-Solanaceae & $\begin{array}{l}\text { Capsicum } \\
\text { frutescens }\end{array}$ & Aucune & Feuilles & 33,33 & $\begin{array}{l}\text { Céphalées, } \\
\text { traumatisme de l'œil }\end{array}$ & $\begin{array}{l}\text { Expression des } \\
\text { feuilles }\end{array}$ & $\begin{array}{l}\text { Une goutte par œil } \\
\text { jusqu'à ce que le mal } \\
\text { finisse }\end{array}$ \\
\hline 60-Malvaceae & Lovoa trichilioides & Aucune & Écorces & 11,11 & Douleur plantaire & $\begin{array}{l}\text {-Trituration des } \\
\text { écorces }\end{array}$ & $\begin{array}{l}\text { Poser les pieds } \\
\text { dessus pendant } 10 \mathrm{mn} \\
\text { matin et soir jusqu'a ce } \\
\text { que la douleur finisse }\end{array}$ \\
\hline 61-Caricaceae & Carica papaya & Aucune & Pepins & 38,88 & dysenterie amibienne & $\begin{array}{l}\text {-Trituration des pépins } \\
\text { de papaye, }\end{array}$ & $\begin{array}{l}1 \text { verre par jour } \\
\text { pendant } 5 \text { jours }\end{array}$ \\
\hline 62-Burseraceae & Dacryodes edulis & Aucune & Feuilles & 35,55 & dysenterie amibienne & -Décoction & $\begin{array}{l}1 \text { verre par jour } \\
\text { pendant } 5 \text { jours }\end{array}$ \\
\hline 63-Fabaceae & $\begin{array}{l}\text { Pterocarpus } \\
\text { soyauxii }\end{array}$ & Aucune & Écorces & 11,11 & Hernie ombicale & -Décoction, filtré & $\begin{array}{l}\text { Se purger matin et soir } \\
\text { jusqu'à ce que la } \\
\text { hernie finisse }\end{array}$ \\
\hline 64-Annonaceae & Annona muricata & Aucune & Feuilles & 25,55 & Fievre typhoide & $\begin{array}{l}\text {-Trituration dans } 1 \\
\text { litre d'eau, filtré. }\end{array}$ & $\begin{array}{l}1 \text { verre par jour } \\
\text { pendant } 5 \text { jours }\end{array}$ \\
\hline 65-Vitaceae & Cissus sp. & Aucune & Feuilles & 27,77 & $\begin{array}{l}\text { Levures chez les } \\
\text { enfants }\end{array}$ & Trituration, filtré & $\begin{array}{l}1 \text { cataplasme matin et } \\
\text { soir pendant } 5 \text { jours }\end{array}$ \\
\hline 66-Fabaceae & Senna hirsuta & Aucune & Feuilles & 13,33 & $\begin{array}{l}\text { Abcès du sein chez la } \\
\text { femme qui allaitent }\end{array}$ & -Trituration & $\begin{array}{l}\text { Matin et soir jusqu'à ce } \\
\text { que l'abcès finisse }\end{array}$ \\
\hline 67-Euphorbiaceae & Manihot esculenta & Aucune & Feuilles & 51 & Anemie & $\begin{array}{l}\text {-Trituration, mélanger } \\
\text { avec du lait non sucrer } \\
\text { +grenadine+jaune } \\
\text { d'œuf, filtrer }\end{array}$ & $\begin{array}{l}\text { La totalité de la } \\
\text { solution est prise matin } \\
\text { et soir. renouveler la } \\
\text { préparation chaque } \\
\text { jour pendant un mois }\end{array}$ \\
\hline 68-Euphorbiaceae & $\begin{array}{l}\text { Ricinodendron } \\
\text { heudelotii }\end{array}$ & Aucune & Feuilles & 30 & Traumatisme de l'oeil & -Expression & $\begin{array}{l}1 \text { goutte par oeil matin } \\
\text { et soir }\end{array}$ \\
\hline 69-Labiaceae & Ocimum viride & Aucune & Feuilles & 30 & $\begin{array}{l}\text { Gingivite, } \\
\text { dermatoses, } \\
\text { helminthiases }\end{array}$ & -Trituration & $\begin{array}{l}\text { Boire } 1 \text { verre par jour } \\
\text { ou se purger une fois } \\
\text { par jour pendant } 4 \\
\text { jours }\end{array}$ \\
\hline 70-Solanaceae & Nicotiana tabacum & Aucune & $\begin{array}{l}\text { Fruits, } \\
\text { feuilles }\end{array}$ & 44,44 & $\begin{array}{l}\text { Insuffisance rénale, } \\
\text { trompes bouchées, } \\
\text { Hémorroïdes }\end{array}$ & $\begin{array}{l}\text {-Décoction ou une } \\
\text { macération }\end{array}$ & $\begin{array}{l}\text { Une purge chaque } \\
\text { après } 2 \text { jours }\end{array}$ \\
\hline
\end{tabular}


Mpondo et al., J. Appl. Biosci. 2017 Connaissances et usages traditionnels des plantes médicinales du département du haut Nyong

\begin{tabular}{|c|c|c|c|c|c|c|c|}
\hline 71-Rutaceae & Citrus limon & Aucune & Fruits & 22,22 & Helminthiases, toux & -Décoction puis filtrer & $\begin{array}{l}1 \text { verre matin et soir } \\
\text { pendant } 5 \text { jours }\end{array}$ \\
\hline 72-Asteraceae & $\begin{array}{l}\text { Ageratum } \\
\text { conyzoides }\end{array}$ & Aucune & $\begin{array}{l}\text { feuilles et } \\
\text { tiges }\end{array}$ & 33,33 & $\begin{array}{l}\text { Fièvre, insuffisance } \\
\text { rénale, hémorroïdes, } \\
\text { ulcère gastrique }\end{array}$ & $\begin{array}{l}\text { Décoction ou } \\
\text { macération }\end{array}$ & \\
\hline 73-Clusiaceae & Hibiscus asper & Aucune & feuilles & 22,22 & toux & - Décoction & 1 verre matin et soir \\
\hline 74-Lauraceae & persea americana & Aucune & $\begin{array}{l}\text { Feuilles, } \\
\text { écorces }\end{array}$ & 44,44 & $\begin{array}{l}\text { Hypercholestérolémie } \\
\text {, ulcère gastrique, } \\
\text { hypertension }\end{array}$ & $\begin{array}{l}\text { - Macération ou } \\
\text { Décoction, filtrer }\end{array}$ & $\begin{array}{l}1 \text { verre matin et soir } \\
\text { pendant } 2 \text { mois }\end{array}$ \\
\hline 75-Malvaceae & Theobroma cacao & Aucune & $\begin{array}{l}\text { Fèves et } \\
\text { cabosses }\end{array}$ & 38,88 & $\begin{array}{l}\text { Dépression nerveuse, } \\
\text { insufisance cardiaque }\end{array}$ & $\begin{array}{l}\text {-Trituration + infusion } \\
+ \text { lait }\end{array}$ & $\begin{array}{l}\text { Boire } 1 \text { verre matin et } \\
\text { soir pendant } 2 \\
\text { semaines }\end{array}$ \\
\hline 76-Myrtaceae & Psidium guajava & Aucune & Feuilles & 35,55 & $\begin{array}{l}\text { dysenterie amibienne, } \\
\text { diarrhée }\end{array}$ & -Décoction & $\begin{array}{l}\text { Boire } 1 \text { verre matin et } \\
\text { soir pendant } 7 \text { jours }\end{array}$ \\
\hline 77-Poaceae & Zea mays & Aucune & Graines & 33,33 & $\begin{array}{l}\text { Infections } \\
\text { cutanées, plaies }\end{array}$ & -Trituration & $\begin{array}{l}\text { Appliquer la pâte sur } \\
\text { l'infection ou la } \\
\text { blessure jusqu'à } \\
\text { cicatrisation }\end{array}$ \\
\hline 78-Musaceae & Musa paradisiaca & Aucune & Fruits & 44,44 & Helminthiases, & -Décoction & \\
\hline 79-Musaceae & Musa sinensis & Aucune & Fruits & 41,11 & $\begin{array}{l}\text { Helminthiases, ulcère } \\
\text { gastrique, diarrhées }\end{array}$ & -Décoction & $\begin{array}{l}\text { Un verre matin et soir } \\
\text { pendant } 1 \text { semaine }\end{array}$ \\
\hline 80-Graminées & $\begin{array}{l}\text { Pennisetum } \\
\text { purpureum }\end{array}$ & Aucune & feuilles & 11,11 & $\begin{array}{l}\text { Microfilaires, mycose } \\
\text { de la peau, gale }\end{array}$ & -Trituration & $\begin{array}{l}\text { faire une friction sur la } \\
\text { peau }\end{array}$ \\
\hline 81-Zingiberaceae & $\begin{array}{l}\text { Aframomum } \\
\text { melegueta }\end{array}$ & Aucune & Fruits & 11,11 & $\begin{array}{l}\text { Insuffisance rénale, } \\
\text { constipation }\end{array}$ & -Décoction & $\begin{array}{l}\text { Matin et soir jusqu'à la } \\
\text { guérison }\end{array}$ \\
\hline 821-Malvaceae & $\begin{array}{l}\text { Abelmoschus } \\
\text { esculentus }\end{array}$ & Aucune & Feuilles & 44,44 & $\begin{array}{l}\text { Ictère, hypertension } \\
\text { artérielle }\end{array}$ & -Infusion & $\begin{array}{l}3 \text { à } 4 \text { verres par jour } \\
\text { avant les repas }\end{array}$ \\
\hline 83-Asteraceae & $\begin{array}{l}\text { Leucanthemum } \\
\text { vulgare }\end{array}$ & Aucune & Feuilles & 50 & Helminthiases & -Décoction & Toute la journée \\
\hline 84-Arecaceae & $\begin{array}{l}\text { xanthosoma } \\
\text { sagitiffilum }\end{array}$ & Aucune & Graines & 33,33 & $\begin{array}{l}\text { Panaris des pieds } \\
\text { Rougeole, } \\
\text { helminthiases }\end{array}$ & -Décoction, filtré & $\begin{array}{l}\text { une purge par jour } \\
\text { selon la gravité de } \\
\text { l'infection de la } \\
\text { rougeole, } 1 \text { verre par } \\
\text { jour pendant } 5 \text { jours }\end{array}$ \\
\hline
\end{tabular}


Mpondo et al., J. Appl. Biosci. 2017 Connaissances et usages traditionnels des plantes médicinales du département du haut Nyong

\begin{tabular}{|c|c|c|c|c|c|c|c|}
\hline 85-Dennstaedtiaceae & $\begin{array}{l}\text { Pteridium } \\
\text { aquilinum }\end{array}$ & Aucune & Feuilles & 27,77 & Ulcères gastriques & $\begin{array}{l}\text {-Macération après } \\
\text { trituration }\end{array}$ & $\begin{array}{l}1 \text { verre matin et soir } \\
\text { pendant } 1 \text { semaine }\end{array}$ \\
\hline 86-Oloaceae & Aloe sp. & Aucune & $\begin{array}{l}\text { Feuilles, } \\
\text { écorces }\end{array}$ & 33,33 & $\begin{array}{l}\text { Constipation, } \\
\text { helminhiases }\end{array}$ & $\begin{array}{l}\text {-Trituration, friction sur } \\
\text { les filaires ou sur les } \\
\text { mycoses }\end{array}$ & $\begin{array}{l}\text { Frotter matin et soir } \\
\text { après le bain }\end{array}$ \\
\hline 87-Asteraceae & $\begin{array}{l}\text { Conyza } \\
\text { sumatrensis }\end{array}$ & Aucune & Feuilles & 11,11 & Microfilaires, mycoses & Trituration & Matin et soir \\
\hline 88-Asteraceae & $\begin{array}{l}\text { Chrysanthemum } \\
\text { coronarium }\end{array}$ & Aucune & \begin{tabular}{|l|} 
Tubercule \\
$\mathrm{s}$ \\
\end{tabular} & 16,66 & Panaris des pieds & Trituration & $\begin{array}{l}\text { poser la pâte sur le } \\
\text { panaris après incision }\end{array}$ \\
\hline 89-Asteraceae & Miltracarpus hirta & Aucune & Feuilles & 13,33 & Mycose superficielle & $\begin{array}{l}\text { Macération pendant } \\
24 \mathrm{~h}\end{array}$ & $\begin{array}{l}\text { boire } 2 \text { verres par jour } \\
\text { jusqu'à ce que le mal } \\
\text { finisse }\end{array}$ \\
\hline $\begin{array}{l}\text { 90-Convolvula } \\
\text { ceae }\end{array}$ & Ipomea batata & Aucune & \begin{tabular}{|l} 
Tubercule \\
$\mathrm{s}$
\end{tabular} & 20 & Constipation & Trituration, filtrer & $\begin{array}{l}\text { Une purge } 2 \text { fois par } \\
\text { jour pendant } 3 \text { jours }\end{array}$ \\
\hline
\end{tabular}




\section{DISCUSSION}

La volonté à fournir des informations par la population était remarquable. Les hommes $(54,55 \%)$ ont plus participé à l'enquête que les femmes (45,55\%). Au Cameroun, Ngoule et al. (2015) ont recensé également une majorité d'hommes $(60,41 \%)$ dans les marchés de Douala Est, tandis que Ndjouondo et al. (2015) ont trouvé un pourcentage élevé de femmes (96,97\%). D'autre part, les enquêtés appartiennent majoritairement à la classe des adultes. Mpondo and Dibong (2012) dans leurs travaux sur les plantes à phénols utilisées par les populations de la ville de Douala ont trouvé des résultats similaires. En outre, les jeunes sont pour la plupart scolarisés et se désintéressent de la médecine traditionnelle, au profit de divers loisirs. Ils rompent ainsi tout contact avec les plantes médicinales et baissent le flux de connaissances entre les adultes et leur génération. Or les connaissances fournies sur les plantes médicinales par les enquêtés ont été majoritairement acquises de leurs ascendants; d'où le risque de disparition des connaissances sur l'utilisation des plantes médicinales en milieu rural en particulier et sur le plan national en général. L'enquête ethnobotanique des plantes médicinales recensées dans trois villages (Djamonomine, Motcheboum, Djende) du département du Haut-Nyong a révélé 90 espèces appartenant à 83 genres et 47 familles. Par contre des résultats différents ont été trouvés au Nigeria par Sofowara (1978) qui a recensé 60 espèces appartenant à 56 genres et 31 familles. Au Botswana, 47 espèces appartenant à 45 genres et 29 familles ont été inventoriées (Setshogo et al., 2010). 58 espèces de 56 genres et 33 familles ont été recensées dans 3 communes de la ville d'Abidjan en Côte d'Ivoire (Trabi et al., 2008). Au Cameroun, des études ethnobotaniques identifient respectivement 30 espèces appartenant à 25 familles et 29 genres et 35 espèces appartenant à 33 genres et 27 familles dans trois marchés de la ville de Douala : Nkolouloun, Dakar et des chèvres (Dibong et al., 2011a). Dans cinq marchés de la ville de Yaoundé, 35 espèces de 35 genres et 18 familles ont été recensées (Betti, 2001). Le nombre d'espèces inventoriées et caractérisées est important et complète les travaux antérieurs non connus déjà effectués dans le département du Haut-Nyong. Par ailleurs, la diversité biologique des sites étudiés est établie comme dans la plupart des milieux forestiers du pays. Cette biodiversité végétale témoigne de la richesse floristique aussi de la richesse en plante médicinale. L'inventaire floristique montre que la famille des Euphorbiaceae et des Asteraceae prédominent. Par contre, au Brésil, une prédominance des Asteraceae et des Lamiaceae est obtenue (Leitao et Rodriguez, 2007). Au Cameroun l'analyse floristique a révélé une prédominance des Fabaceae et des Asteraceae (Ngene et al., 2015). Une prédominance de Fabaceae, suivie d'Asteraceae, d'Apocynaceae et d'Euphorbiaceae est observée dans les caractéristiques floristiques des espèces à flavonoïdes recensées dans les marchés de la ville de Douala (Kidik et al., 2015). Les espèces afro tropicales sont les citées (34,44\%). Cette dominance d'espèces afro tropicales traduit ainsi un fort impact des cultures étrangères sur l'exploitation des plantes médicinales et la répartition des cultures locales (Betti, 2002). Par contre Betti montre que la ville de Bertoua et de Yaoundé ont une faible proportion des espèces étrangères $(23,76 \%)$, mais plutôt une importante domination des espèces du domaine guinéen $(67,54 \%)$. Les espèces Guinéocongolaises sont nombreuses (22\%); les travaux effectués par Kidik sur la caractérisation des plantes médicinales à flavonoïdes des marchés de Douala (Cameroun). Ngoule et al. (2015) montrent que les espèces pantropicales et guinéo-congolaises $(71 \%)$ sont les plus représentées. Les types morphologiques observés témoignent d'une majorité des espèces ligneuses (arbres) ce qui concorde avec une étude qui a recensé $60 \%$ de ligneux contre $40 \%$ d'herbacées (Dibong et al., 2011b). Des résultats similaires sont retrouvés dans une étude ethnobotanique des plantes médicinales vendues dans le marché d'Abomey-Calavi au Bénin (Adomou et al., 2012). Les espèces ligneuses sont sollicitées dans la plupart du temps pour leur écorce ou leurs racines et l'exploitation de ces organes peut s'avérer préjudiciable pour la plante et même pour son écosystème. En effet, outre la quantité du produit à exploiter, la gravité de cette vulnérabilité varie en règle générale, en fonction de la technique de prélèvement. En effet, cette vulnérabilité ne se limite pas seulement à la technique de prélèvement et aux quantités du produit à exploiter mais aussi à la forte poussée de la déforestation qui sévit dans le département du Haut-Nyong en particulier et au Cameroun en général. Par ailleurs, la strate herbacée est constituée des espèces de petite taille, présentant une maturité précoce, une descendance nombreuse et une capacité de régénération très rapide leur permettent d'être tout le temps disponibles, et réduit ainsi la pression sur les écosystèmes (Cunningham, 1996). Les plantes médicinales inventoriées totalisent 5 types de diaspores. Les sarcochores sont les plus représentés. Leur mode de dissémination est la zoochorie ce qui favorise l'extension des espèces et la diversification de son patrimoine génétique. Autrement 
dit, la faune joue un rôle primordial dans la régénération de la plupart des plantes (Ngankoue, 2013). Dans le Dja, l'importance des espèces zoochores en zone forestière sur les plantes médicinales a été démontrée (Betti, 2002). Une étude montre également que le mode de dissémination le plus répandu est la zoochorie avec $71 \%$ (Ngankoue, 2013). Le mode de préparation le plus pratiqué est la décoction $(38,04 \%)$. la population locale croit au mode de décoction et le trouve adéquat pour réchauffer le corps et désinfecter la plante (Bwassiwe et al., 2014). D'autre part, la décoction permet de recueillir le plus de principes actifs et atténue ou annule l'effet toxique de certaines recettes. Ce pourcentage est proche de $42 \%$ selon une étude de screening phytochimique sur quelques plantes en Côte d'lvoire (N'guessan et al., 2009). II est très élevé par rapport à $33 \%$ selon la contribution aux études ethnobotaniques des plantes de la République Populaire du Bénin (Adjanohoun et al., 1989). Les informateurs ignorent les poids et les mesures précises dans la préparation et la posologie des phytomédicaments. La précision fait défaut sur plusieurs plantes comme les quantités d'organes végétaux à préparer, le solvant ou le véhicule utilisé, le temps nécessaire à la préparation des solutions (décoction, infusion, macération et trituration) et la dose précise à prescrire. La dose peut être par poignée, par cuillerée ou par pincée. La dose reste encore aléatoire.Seulement deux plantes recensées montrent une fréquence de citation supérieure ou égale à $50 \%$, Euphorbia hirta et Manihot esculenta avec chacune $51 \%$. Autrement dit, ces deux plantes sont majoritairement utilisées pour soigner les maladies dans un même village ou entre les villages enquêtés. Pour des maladies communes, ces plantes constituent des atouts dans la mise en œuvre de la pharmacopée traditionnelle du département du HautNyong et des recherches ethnopharmacologiques pousser pourrait aboutir à l'obtention des médicaments traditionnels améliorés (MTA) efficaces dans le traitement des maladies concernées. La connaissance traditionnelle des pratiques médicinales est donc répandue parmi les populations du Haut-Nyong et doivent être poursuivie et documentée pour être révéler à d'autres populations de la forêt vivant en harmonie avec l'environnement (Bhat and Jacob, 1995). La culture scientifique, la conservation et l'usage durable des espèces végétales par les communautés ethnies seraient largement avantageux pour la croissance socio-économique dans la conservation des espèces rares et la connaissance indigène pour les générations futures (Birendra and Chhetri, 2009). Abong-Mbang n'a pas de marché médicinal, le marché le plus proche est à Bertoua cheflieu de la région de l'Est. De plus, les herboristes le fréquentent périodiquement. La pression sur le potentiel en plante médicinales est donc faible et le milieu forestier environnant est moins dégradé par ses activités entreprises. L'indice de consensus des informateurs (ICFs) est supérieur à 0,5 pour 15 catégories de maladies recensées sur les 22 catégories connues par l'OMS en 2011 , soit $72,72 \%$ de chance pour qu'un habitant de ce département souffre de ces maladies dans ce département. La santé des populations est relativement bonne ce qui diminue la pression sur les ressources en plantes médicinales. Cependant, cinq catégories de maladie ont un ICFs supérieur ou égale à 0,5 et les plantes utilisées pour leur traitement ont une pression élevée. Leur potentiel mériterait donc d'être entretenu pour éviter que ces espèces se trouvent menacées et placées dans la liste rouge de l'organisme qui s'occupe des espèces menacées. Par ailleurs les médicaments à base de plante apportent des réponses thérapeutiques dans de nombreuses indications, limitant les maladies iatrogènes. Les feuilles et les écorces sont les parties de la plante les plus sollicitées par les enquêtés. La préférence pour ces organes végétatifs s'explique par leur facilité à être récoltée (Brunneton, 1999 ; Trabi et al., 2008). Toutefois, sa fréquente serait justifiée par l'abondance des groupes chimiques qu'elles contiennent, car connues comme le lieu de synthèse des métabolites secondaires du végétal. La variabilité spécifique observée et des organes utilisés démontrent la possibilité d'obtention d'une grande variété de molécules biologiques entrant dans la phytothérapie, notamment l'aromathérapie. Par ailleurs, les utilisateurs des plantes ont tendance à arracher la plante entière au lieu de s'intéresser uniquement à la partie souhaitée (principalement les feuilles). D'autre part, il existe une relation manifeste entre la partie utilisée de la plante exploitée et les effets de cette exploitation sur son existence, compromettant sérieusement la durabilité des espèces médicinales surtout les bulbeuses (Betti et Mebere, 2011). Autrement dit, cette partie de la plante est importante, car elle est le siège de la photosynthèse et parfois du stockage des métabolites secondaires responsables des propriétés biologiques de la plante (Ngono et al., 2011). Le prélèvement de $50 \%$ des feuilles d'un arbre n'affecte pas de façon significative sa survie (Ouattara, 2006). Par ailleurs, l'intérêt porté aux écorces relève de leur richesse en métabolites secondaires responsables des propriétés biologiques de la plante (N'Guessan et al., 2009). 


\section{CONCLUSION}

L'enquête ethnobotanique et ethnopharmacologique menée a permis de dresser un catalogue de 90 espèces médicinales. Ces taxons sont répartis en 47 familles et 83 genres avec une nette dominance de la famille des Euphorbiaceae. Les ligneux prédominent par rapport aux herbacées. Les espèces afrotropicales sont les plus représentées et les sarcochores le type le plus répandu. La plupart des espèces médicinales sont très utilisées dans le traitement des maladies infectieuses intestinales, des maladies dues aux protozoaires et les symptômes. Ces maladies sont traitées surtout par les feuilles qui

\section{REFERENCES}

Adjanohoun $E$, Adjakidjè $V$, Ahyi $M$, Aké Assi L, Akoègninou $A$, d'Almeida $J$ et al. 1989. Contribution aux Etudes Ethnobotaniques et Floristiques en République Populaire du Bénin. Agence de Coopération Culturelle et Techniques Paris. $895 \mathrm{p}$.

Adomou A, Yedomonhan $H$, Djossa B, Legba $S$, Oumorou M, Akoegninou A. 2012. Étude Ethnobotanique des plantes médicinales vendues dans le marché d'Abomey-Calavi au Bénin. Int. J. Biol. Chem. Sci. 6: 745-772.

Bath R, Jacobs T. 1995. Traditional herbal medicine in Transkei. Journal of ethnopharmacology 48: 712.

Betti J. 2001. Usages traditionnels et vulnérabilité des plantes médicinales dans la réserve de biosphère du Dja et dans les marchés de Yaoundé, Cameroun. Thèse Doctorat, Université Libre de Bruxelles, Belgique. 289p.

Betti J. 2002. Medicinal plants sold in Yaoundé markets, Cameroon. African Study Monographs 23: 4764.

Betti J, Mebere S. 2011. Contribution à la connaissance des produits forestiers non ligneux du parc national de Kalamaloué, Extrême Nord Cameroun: les plantes alimentaires. International Journal of Biological and Chemical biosciences 5 (1) : 291 - 303.

Birendra M, Chhetri R. 2009. Indigenous knowledge on ethnobotanical plants of Kavrepalanchowk district. Journal of Science, Engineering and Technology 5: 96 - 109.

Brunneton J. 1999. Pharmacognosie, phytochimie, plantes médicinales, 3ieme édition. Éditions médicinales internationales, éditions Tec et Doc Lavoisier. $1120 \mathrm{p}$. constituent les organes végétaux les plus utilisés et la décoction représente le mode de préparation le plus dominant. Les effets thérapeutiques induits par les plantes, pour traiter les pathologies sont le fait de divers groupes chimiques: alcaloïdes, tanins, flavonoïdes, polyphénols, qui constituent la base scientifique de l'utilisation thérapeutique traditionnelle des plantes utilisées. Ces propriétés thérapeutiques citées peuvent justifiées l'intégration des plantes médicinales dans le programme d'aménagement forestier, en vue de leur exploitation durable.

Bwassiwe H, Metowogo K, Aklesso P, Mouzou R, Tossou R, Ahounou J, Eklu-Gadegbekou K, Dansou P, Aklikokou k. 2014. Enquête ethnobotanique sur les plantes utilisées dans le traitement traditionnel des contusions musculaires au Togo. $24: 112-130$.

Cunningham A. 1996. Peuples, parc et plantes. Recommandations pour les zones à usages multiples et les alternatives de développement autour du parc national de Bwindi Impénétrable, Ouganda. Documents de travail Peuples et Plantes $n^{\circ}$ 4. UNESCO, Paris. $66 p$.

Dibong S, Mpondo M, Ngoye A, Kwin N, Betti JL. 2011a. Ethnobotanique et phytomédecine des plantes médicinales vendues sur les marchés de Douala Cameroun. Journal of Applied Biosciences 37: $2496-2507$.

Dibong S, Mpondo M, Ngoye A, Kwin M, Betti J. 2011b. Ethnobotanique et phytomédecine des plantes médicinales vendues sur les marchés de Douala, Cameroun. Journal of Applied Biosciences 37: 2496-2507.

Kanta V, Unnati S, Ritu M. 2011. A review on aids and herbal remedies. International Journal of Research in Ayurveda \& Pharmacy 2 (6): 17091713.

Kidik P, Ngene J, Ngoule C, Mvogo O, Ndjib R, Dibong SD, Mpondo Mpondo E. 2015. Caractérisation des plantes médicinales à flavonoïdes des marchés de Douala (Cameroun). Int. J. Biol. Chem. Sci. 9 (3): 1494 - 1516.

Leitao, Rodriguez E. 2007. Plants of restricted use indicated by three cultures in Brazil. Cabocloriver dweller, Indian and Quilombola. Journal of Ethnopharmacology 111: 295 - 302.

Mpondo Mpondo E, Dibong SD. 2012. Traditional knowledge on medicinal plants use by ethnic 
communities in Douala, Cameroun. European Journal of Medicinal Plants 2(2): 159-176.

Muthu C, Ayyanar M, Raja N, Ignacimuthu S. 2006. Medicinal plants used by traditional healers in Kancheepuram District of Tamil Nadu, India. Journal of Ethnobiology and Ethnomedicine 2: 1746-4269.

Ngene J, Ngoule C, Kdik P, Mvogo O, Ndjib R, Dibong SO, Mpondo Mpondo E. 2015. Importance dans la pharmacopée traditionnelle des plantes à flavonoïdes vendues dans les marchés de Douala est (Cameroun). Journal of Applied Biosciences 88 : 8194 - 8210.

Newall C, Anderson L, phillipson J. 1996. A guide for health-care professional. The pharmaceutical press, London, herbal medicines ; 1-296.

Ndjouondo G, Ngene J, Ngoule C, Kidik P, Ndjib R, Dibong SD. 2015. Inventaire et caractérisation des plantes médicinales des sous bassins versants Kambo et Longmayagui (Douala, Cameroun). Journal of Animal et Plant Sciences 25 (3): 3898-3916.

Ngankoue M. 2013. Caractéristiques floristiques et importance économique des produits forestiers non ligneux alimentaires. Mémoire de DEA, Université de Douala. $60 \mathrm{p}$.

Ngono N, Koanga M, Tchinda T, Magnifouet, Motso C, Mballa B. 2011. Etnobotanical survey of some camerounian plants used for treatment of viral diseases. African Journal of Plant Science 5 (1): 15-21.

N'Guessan K, Kadja B, Zirihi G, Traoré D, Aké-assi L. 2009. Screening phytochimique de quelques plantes médicinales ivoiriennes. Sciences \& Nature 6: 1 - 15.

Ngoule C, Ngene J, Kidick P, Ndjib R, Dibong S, Mpondo Mpondo E. 2015. Inventaire et caractérisation floristiques des plantes médicinales à huiles essentielles des marchés de Douala Est (Cameroun). Int. J. Biol. Chem. Sci. 9(2): 874889.

Ouattara D. 2006. Contribution à l'inventaire des plantes médicinales significatives utilisées dans la région de Divo (sud forestier de la Côte-d'Ivoire) et à la diagnose du poivrier de Guinée : Xylopia aethiopica (Dunal) A. Rich. (Annonaceae). Thèse de Doctorat de l'Université de CocodyAbidjan, Côted'Ivoire. 184p.

Patwardhan B. 2005. Ethnopharmacology and drug discovery. Journal of Ethnopharmacology 100 : 50-52.
Setshogo M, Mbereki M. 2010. Floristic diversity and uses of medicinal plants sold by Street vendors in Gaborone, Botswana. African Journal of Plant Science and Biotechnology 5 (1): 69 -74.

Sofowara F. 1978. Antimicrobial alkaloids from Nigerian chewing stick (TagaroZanthoxyloides). Plant med. 36: 2004 - 2007.

Trabi F, Irié G, N'gaman K, Clejesson H, Mohou. 2008. Études de quelques plantes thérapeutiques utilisées dans le traitement de l'hypertension artérielle et du diabète : deux maladies émergentes en Côte d'lvoire. Sciences et Nature 5 (1): $39-48$.

World Health Organization. Family of International Classifications. Consulté le 12 juillet 2011. 